BRAZIULIAN JOURNAL

OF MEDICAL AND BIOLOGICAL RESFARCH

www.bjournal.com.br
ISSN 0100-879X

Volume 44 (11) 1070-1193 November 2011

BIOMEDICAL SCIENCES

AND

CLINICAL INVESTIGATION

Braz J Med Biol Res, November 2011, Volume 44(11) 1171-1176

doi: 10.1590/S0100-879X2011007500135

\title{
Identification of sarcopenic obesity in postmenopausal women: a cutoff proposal
}

R.J. Oliveira, M. Bottaro, J.T. Júnior, P.T.V. Farinatti, L.A. Bezerra and R.M. Lima

The Brazilian Journal of Medical and Biological Research is partially financed by

\section{Q Q}

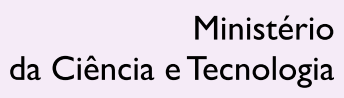

Ministério da Educação

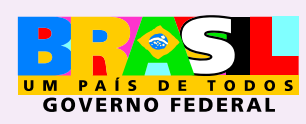

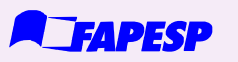

Institutional Sponsors
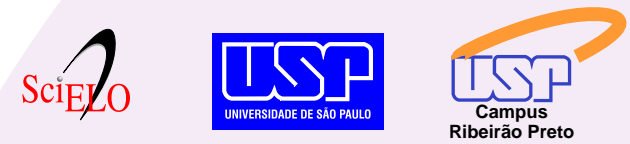
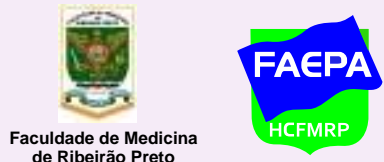

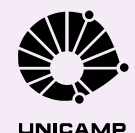

UNICAMP
Ф SHIMADZU

Explore High - Performance MS Orbitrap Technology In Proteomics \& Metabolomics

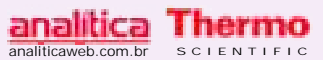




\title{
Identification of sarcopenic obesity in postmenopausal women: a cutoff proposal
}

\author{
R.J. Oliveira ${ }^{1}$, M. Bottaro' ${ }^{1}$, J.T. Júnior ${ }^{2}$, P.T.V. Farinatti ${ }^{3}$, \\ L.A. Bezerra ${ }^{2}$ and R.M. Lima ${ }^{1}$ \\ ${ }^{1}$ Faculdade de Educação Física, Universidade de Brasília, Brasília, DF, Brasil \\ ${ }^{2}$ Programa de Pós-Graduação em Educação Física, Universidade Católica de Brasília, Brasília, DF, Brasil \\ ${ }^{3}$ Laboratório de Atividade Física e Promoção da Saúde, Universidade do Estado do Rio de Janeiro, \\ Rio de Janeiro, RJ, Brasil
}

\begin{abstract}
Sarcopenic obesity is the combination of reduced fat-free mass (FFM) and increased fat mass (FM) with advancing age but there is lack of clear criteria for its identification. The purposes of the present investigation were: 1) to determine the prevalence of postmenopausal women with reduced FFM relative to their FM and height, and 2) to examine whether there are associations between the proposed classification and health-related variables. A total of 607 women were included in this cross-sectional study and were separated into two subsets: 258 older women with a mean age of $66.8 \pm 5.6$ years and 349 young women aged 18-40 years (mean age, $29.0 \pm 7.5$ years). All volunteers underwent body composition assessment by dual-energy X-ray absorptiometry. The FFM index relative to FM and height was calculated and the cutoff value corresponded to two standard deviations below the mean of the young reference group. To examine the clinical significance of the classification, all older participants underwent measurements of quadriceps strength and cardiorespiratory fitness. Values were compared between those who were classified as low FFM or not, using an independent samples $t$-test and correlations were examined. The cutoff corresponded to a residual of -3.4 and generated a sarcopenic obesity prevalence of $19.8 \%$ that was associated with reduced muscle strength and aerobic fitness among the older participants. Also, the index correlated significantly with the health-related fitness variables. The results demonstrated reduced functional capacity for those below the proposed cutoff and suggested applicability of the approach as a definition for sarcopenic obesity.
\end{abstract}

Key words: Fat-free mass; Sarcopenia; Aging; Muscle strength

\section{Introduction}

Aging is a continuous process characterized by a decline in most physiological functions. Alterations that are widely recognized by gerontologists include the decline in both fat-free mass (FFM) and muscle strength, a process that has been referred to as sarcopenia (1). The term sarcopenia comes from the Greek words sarx (flesh) and penia (deficiency) and was originally proposed to refer to the involuntary loss of muscle mass that commonly occurs with advancing age (1-4). This phenomenon has been described in both elderly men and women (2) and has been linked to multiple negative clinical outcomes (24). Sarcopenia imposes an important economic onus on health care costs (5), and thus efforts to understand and counteract this condition are meaningful. Of note, it has been argued that since women have less muscle mass compared to men, they may be at higher risk for functional limitations and disability (4).

Although the decline in muscle strength and mass is well known to have a negative effect on function and autonomy among older individuals, lack of clear criteria for classification of sarcopenia leads to limitations in clinical practice and when performing epidemiological studies $(4,6)$. Dualenergy X-ray absorptiometry (DXA) has become a practical technique for measuring total body FFM and fat mass (FM) in clinical and research settings $(2-4,6)$. Moreover, DXA permits measurements of FFM and FM for the trunk, arms, and legs. The sum of arm and leg FFM has been named appendicular FFM (AFFM), and this measure has been demonstrated to consistently correlate with muscle mass in humans across the age span $(7,8)$. DXA is a useful method for distinguishing fat, bone mineral and lean tissues $(2,6,9)$ and thus has an important potential for assessment

Correspondence: R.M. Lima, Faculdade de Educação Física, UnB, Campus Universitário Darcy Ribeiro, 70910-900 Brasília, DF, Brasil. Fax: +55-61-3107-2500. E-mail: ricardomoreno@unb.br

Received November 15, 2010. Accepted September 29, 2011. Available online October 14, 2011. Published November 14, 2011. 
of age-associated changes in body composition.

Height is a variable that is positively correlated with total FFM (2). In this respect, relative FFM has been frequently defined as AFFM divided by height squared, analogous to the use of body mass index $(2,10)$. In the New Mexico Aging Process Study (2), cutoff values for sarcopenia were defined as relative AFFM two standard deviations below reference data of young adults aged 18-40 years, similar to the method used to define osteoporosis. This approach showed that deficiency in muscle mass increases with age and the prevalence of sarcopenia is associated with a significantly higher risk of disability in elderly individuals. However, in addition to the decline in FFM, older individuals also tend to gain weight, especially fat mass, as they age (3). Therefore, an expanding literature in this area has been focusing on a high FM/low FFM combination (4), a condition referred to as sarcopenic obesity (11-13). The combination of low muscle strength and obesity, a state named dynapenic-obesity, has been associated with poorer physical function than obesity alone (11), and thus has been recently examined as an important cause of frailty among older adults $(12,13)$. In contrast, data reported by Bouchard et al. (14) have suggested that obesity per se contributes more to a lower physical capacity than sarcopenia, indicating that additional studies are warranted to better understand this phenomenon.

Newman et al. (4) highlighted the importance of considering body FM when examining sarcopenia. They showed that, without contemplating FM, obese individuals may not appear to be sarcopenic even though their muscle mass may be reduced for their body size. These investigators (4) proposed an approach to determine low FFM accounting for FM that was shown to be related to functional limitations and to markers of inflammation (15) in older individuals. However, the importance of determining its association with other health-related outcomes such as muscle strength and function has been highlighted. In addition, Newman et al. (4) did not establish a cutoff value below which individuals were classified as low FFM for their body size. Therefore, the purposes of the present investigation were: 1) to calculate the prevalence of postmenopausal women with reduced FFM relative to their FM and height (i.e., sarcopenic obesity), and 2) to examine whether there are associations between the proposed classification and health-related variables.

\section{Material and Methods}

\section{Participants}

A total of 607 women from a program developed at Universidade Católica de Brasília were included in this cross-sectional study. The sample consisted of the two following subsets: 1) older women with a mean age of 66.8 years $(N=258)$ who were all postmenopausal, did not have metallic prosthesis implants or artificial pacemakers, were not smokers, did not undergo hip replacement surgery, could walk without assistance, and did not have any metabolic or endocrine disorder known to affect the musculoskeletal system, and 2) a dataset of 349 young women aged 18-40 years (mean age 29.0 years) with the same exclusion criteria who were used as reference values to define cutoff values for low FFM. All volunteers answered face-to-face questionnaires addressing medical history, hormone replacement therapy, lifestyle habits, and medication use.

After a brief verbal communication covering the objectives, procedures involved, and possible risks and benefits of the study protocol, written informed consent was obtained from each participant. The study design and procedures were approved by the Universidade Católica de Brasíia Ethics Committee (protocol \#024/2007).

\section{Anthropometry and body composition}

Body weight was measured in the fasting state to the nearest $0.1 \mathrm{~kg}$ using a calibrated electronic scale with volunteers dressed in light T-shirt and shorts. Height was determined without shoes to the nearest $0.1 \mathrm{~cm}$ using a wall stadiometer, after a voluntary deep inspiration. Body mass index (BMI) was calculated as body weight divided by height squared $\left(\mathrm{kg} / \mathrm{m}^{2}\right)$.

Body composition measurements were obtained at the Image Laboratory of the Universidade Católica de Brasília using DXA (DPX-L, Lunar Radiation Corporation, USA). All measurements were carried out by the same trained technician according to procedures described elsewhere (3). AFFM was calculated as the sum of FFM for both arms and legs. In addition, AFFM was considered to be relative to body height squared $\left(\mathrm{kg} / \mathrm{m}^{2}\right)$. The coefficients of variation observed for DXA were 2.1 and $1.9 \%$ for FM and FFM, respectively.

\section{Definition of sarcopenic obesity}

To account for the potential influence of FM on FFM, a measure of relative FFM was calculated according to the specifications of Newman et al. (4). Briefly, this measure is calculated by using a linear regression that predicts each volunteer's AFFM from height (in meters) and whole-body fat mass (in $\mathrm{kg}$ ). The difference between the regressiongenerated prediction and the actual measured AFFM (i.e., residual) represents the objective value. After measuring relative FFM, the cutoff value for the present study was calculated. Women with values equal to or below two standard deviations of the mean of the reference sample consisting of young women were classified as having reduced FFM for their body size. The linear regression generated the following equation: AFFM $=-14,529+(17,989 x$ height in meters $)+(0.1307 \times$ total fat mass in $\mathrm{kg})$.

Subsequently, analyses of the young women's values revealed that two standard deviations below the mean was a residual of -3.4 . Therefore, older women who presented a residual equal to or below this value were considered to have 
low FFM for their body size (i.e., sarcopenic obesity).

\section{Health-related phenotype measurements}

To examine the clinical significance of the proposed cutoff value, all older participants underwent quadriceps muscle strength assessment and a symptom-limited cardiopulmonary exercise test. Both laboratory tests were conducted according to the procedures described below.

Isokinetic muscle strength. Dominant knee extensor isokinetic peak torque was evaluated using the Biodex System 3 dynamometer (Biodex Medical System, USA) (16) as previously described (3). Briefly, the testing protocol consisted of three sets of four knee extensor contractions at $60 \%$ with a $30-\mathrm{s}$ rest between sets (17). The recorded value was the single muscle contraction that elicited the highest peak torque (PT) throughout the protocol, which is expressed in absolute values $(\mathrm{Nm})$ as well as relative to body weight and FFM $(\mathrm{Nm} / \mathrm{kg})$. The equipment was calibrated according to manufacturer specifications before every testing session (18).

Cardiopulmonary exercise test. A total of 176 older volunteers underwent a graded cardiopulmonary exercise test on a treadmill (model RT 300, Moviment, Brazil) until voluntary exhaustion. Before testing, volunteers were prepared with a three-lead electrocardiogram and were given basic instructions related to the procedures and use of the Borg scale for the rating of perceived exertion. Subjects were encouraged to exercise until they felt unable to continue. The rate of exercise intensity increments was based on a ramp protocol, which was designed for exhaustion achievement between 8 and $12 \mathrm{~min}$. The protocol consisted of an initial speed and grade of $2 \mathrm{~km} / \mathrm{h}$ and $0 \%$, respectively, programmed to reach $6 \mathrm{~km} / \mathrm{h}$ and $6 \%$ in $10 \mathrm{~min}$. Blood pressure was measured every 3 min with a mercury sphygmomanometer. Throughout the test, expired gases were continuously measured on a "breath-by-breath" basis (Cortex Metalyzer 3B, Cortex Biophysik, Germany). Volume and gas calibration were performed before each test. All tests were conducted under physician supervision and volunteers were allowed to hold on to the front treadmill handrail. Peak $\mathrm{VO}_{2}$ was calculated as the mean $\mathrm{VO}_{2}$ during the last minute of the test. Besides peak $\mathrm{VO}_{2}$, ventilatory threshold (VT) was also determined. The VT was the point at which there was a loss of linearity between oxygen uptake and carbon dioxide production or the point where the ventilatory equivalent for oxygen or end-tidal oxygen partial pressure curves reached their respective minimum values and began to rise during the progressive exercise test $(19,20)$. VT was determined by two proficient exercise physiologists.

\section{Statistical analyses}

Baseline data are reported as means \pm SD. Independent samples $t$-tests were used to compare variables between young and older women. Using the cutoff value proposed in this study (i.e., a residual equal to or below -3.4), the older women were classified as low FFM or not, and health-related variables were compared among them using an independent samples $t$-test. Also, the relationship between the residuals generated by the equation with muscle strength and aerobic fitness indexes was examined using the Pearson correlation coefficient. Data were considered to be significant at $P<0.05$ and statistical analyses were performed using the SPSS 15.0 software (SPSS, USA).

\section{Results}

The physical characteristics of the whole sample and of the subgroups consisting of young or older women are displayed in Table 1. The older volunteers presented significantly lower height and greater BMI and percent body fat values compared to the young reference group. Absolute and relative FFM were significantly lower in the older subjects; however, total FFM did not reach statistical significance when comparing young vs older women.

Using the proposed cutoff value (i.e., a residual of -3.4), the older women were classified as having low FFM or not, and health-related phenotypes were compared (Table 2). Volunteers classified as having sarcopenic obesity presented lower values of muscle strength, represented by both absolute and relative $\mathrm{PT}(\mathrm{P}=0.019$ and $<0.001$, respectively). Mean aerobic capacity indexes were greater for subjects who were not below the cutoff value, although statistical significance was observed only for absolute $\mathrm{VO}_{2}$ at VT $(\mathrm{P}=0.047)$, time to VT $(\mathrm{P}=0.033)$, and peak oxygen consumption $(P=0.015)$. In addition, women classified as low FFM had higher height $(P<0.001)$ and

Table 1. Physical characteristics of the subjects as a whole and of the subgroups composed of young or older women.

\begin{tabular}{lccc}
\hline & Total sample & Young women & Older women \\
\hline $\mathrm{N}$ & 607 & 349 & 258 \\
Age $($ years $)$ & $44.8 \pm 19.9$ & $29.0 \pm 7.5$ & $66.8 \pm 5.6$ \\
Weight $(\mathrm{kg})$ & $62.3 \pm 12.1$ & $59.8 \pm 11.9$ & $65.7 \pm 11.7^{*}$ \\
Height $(\mathrm{m})$ & $1.57 \pm 0.07$ & $1.60 \pm 0.06$ & $1.53 \pm 0.07^{\star}$ \\
BMI $\left(\mathrm{kg} / \mathrm{m}^{2}\right)$ & $25.4 \pm 5.0$ & $23.5 \pm 4.5$ & $28.0 \pm 4.5^{\star}$ \\
Body fat $(\%)$ & $35.6 \pm 8.1$ & $32.7 \pm 8.2$ & $39.6 \pm 6.0^{*}$ \\
Total FFM $(\mathrm{kg})$ & $38.0 \pm 4.8$ & $38.2 \pm 4.5$ & $37.7 \pm 5.2$ \\
AFFM $(\mathrm{kg})$ & $15.8 \pm 2.6$ & $16.8 \pm 2.5$ & $14.5 \pm 2.2^{*}$ \\
Relative AFFM $\left(\mathrm{kg} / \mathrm{m}^{2}\right)$ & $6.4 \pm 0.8$ & $6.6 \pm 0.8$ & $6.2 \pm 0.8^{*}$ \\
\hline
\end{tabular}

$\mathrm{BMI}=$ body mass index; FFM = fat-free mass; AFFM = appendicular fat-free mass (corresponds to the sum of arms and legs fat-free mass); relative AFFM corresponds to appendicular fat-free mass divided by height squared. ${ }^{*} P<0.05$ compared to young women (independent samples $t$-test). 
Table 2. Health-related physical fitness variables according to classification of sarcopenic obesity using the cutoff value proposed in the present study.

\begin{tabular}{lcc}
\hline & Sarcopenic obesity & Non-sarcopenic obesity \\
\hline $\mathrm{N}(\%)$ & $49(19.8 \%)$ & $198(80.2 \%)$ \\
Age (years) & $67.0 \pm 5.7$ & $66.5 \pm 5.5$ \\
Weight $(\mathrm{kg})$ & $68.7 \pm 11.4$ & $65.1 \pm 11.5$ \\
Height $(\mathrm{m})$ & $1.57 \pm 0.05$ & $1.52 \pm 0.06^{*}$ \\
$\mathrm{BMI}\left(\mathrm{kg} / \mathrm{m}^{2}\right)$ & $28.0 \pm 4.4$ & $28.0 \pm 4.5$ \\
Percent body fat $(\%)$ & $43.3 \pm 4.3$ & $38.6 \pm 6.0^{*}$ \\
$\mathrm{PT}(\mathrm{Nm})$ & $87.5 \pm 17.8$ & $96.0 \pm 23.4^{*}$ \\
$\mathrm{PT}$ relative to body weight $(\mathrm{Nm} / \mathrm{kg})$ & $1.3 \pm 0.2$ & $1.5 \pm 0.3^{*}$ \\
Relative $\mathrm{VO}{ }_{2}$ at $\mathrm{VT}\left(\mathrm{mL} \cdot \mathrm{kg}^{-1} \cdot \mathrm{min}^{-1}\right)$ & $12.1 \pm 2.3$ & $12.7 \pm 2.5$ \\
Absolute $\mathrm{VO} \mathrm{O}_{2}$ at $\mathrm{VT}\left(\mathrm{L} / \mathrm{min}^{*}\right)$ & $0.8 \pm 0.2$ & $0.8 \pm 0.2^{*}$ \\
Time at $\mathrm{VT}(\mathrm{s})$ & $261.6 \pm 89.4$ & $299.4 \pm 97.1^{*}$ \\
Peak relative $\mathrm{VO} \mathrm{O}_{2}\left(\mathrm{~mL} \cdot \mathrm{kg}^{-1} \cdot \mathrm{min}^{-1}\right)$ & $16.5 \pm 2.8$ & $17.3 \pm 3.3$ \\
Peak absolute $\mathrm{VO} 2\left(\mathrm{~L} / \mathrm{min}^{*}\right)$ & $1.0 \pm 0.2$ & $1.1 \pm 0.3^{*}$ \\
Time to exhaustion $(\mathrm{s})$ & $566.2 \pm 124.0$ & $590.7 \pm 125.5$ \\
\hline
\end{tabular}

$\mathrm{BMI}=$ body mass index; $\mathrm{PT}=$ peak torque $; \mathrm{VO}_{2}=$ oxygen consumption; $\mathrm{VT}=$ ventilatory threshold. The cutoff value was two standard deviations below the mean of the young reference group and corresponded to a residual of -3.4 . ${ }^{*} \mathrm{P}<0.05$ compared to sarcopenic obesity (independent samples $t$-test).

percent body fat $(P<0.001)$ values. Overall, health-related fitness variables were unfavorable for those classified with sarcopenic obesity. Reinforcing these observations, the residuals of the equation were significantly correlated with absolute $(r=0.46 ; P<0.001)$ and relative $(r=0.41 ; P<$ $0.001) \mathrm{PT}$, and with oxygen uptake both at VT $(r=0.22$; $\mathrm{P}$ $=0.005)$ and at peak effort $(r=0.29 ; P<0.001)$.

\section{Discussion}

The main contribution of the present study is the proposal of a sarcopenic obesity cutoff value below which postmenopausal women would be classified as having low FFM for their height and fat mass. To examine the possible clinical significance of such classification, health-related fitness variables were measured in the elderly sample and values were compared between low relative FFM and normal relative FFM subgroups. The results clearly pointed to an unfavorable profile for those below the proposed cutoff, specifically exhibiting lower muscle strength and aerobic capacity values. To our knowledge, no previous studies have developed a cutoff value for classifying older women as having low FFM for their body size and the present observations argue in favor of the ability to detect functional consequences. Obviously, future investigations are necessary to better examine whether this approach might be helpful for the screening of individuals who would benefit from interventions designed to increase muscle mass, strength, and aerobic fitness, and to decrease fat mass. The relevance of a cutoff value becomes important when considering the fact that DXA is commonly used in older women but the results are generally limited to the interpretation of bone mineral density. Nevertheless, it is also relevant for future studies to develop methods for sarcopenic obesity identification based on low costs and easily available tools such as tapes, weight scale, stadiometer, or skinfold caliper, in case of inaccessibility to DXA.

An increasing number of studies have been conducted to better understand the pathophysiology of sarcopenia $(2,3,6,21)$, but recent literature has also focused on the obesity/low muscle mass combination (22-27) with sarcopenic obesity described as an important public health problem $(24,26)$. Initial studies proposed the clinical detection of sarcopenic obesity by bioelectrical impedance analysis (27) but later reports emphasized the importance of using more sophisticated measurements such as DXA through clinical examination (22). Although Newman et al. (4) had considered FM and height in their approach, they did not use the term sarcopenic obesity in their report. However, we argue that their method can be used when studying the obesity/low muscle mass combination. In the present study, we explored the Newman et al. (4) approach to define a cutoff value, which was a residual equal to or below -3.4, corresponding to at least two standard deviations below the mean presented by 349 young healthy women aged 18-40 years, analogous to the study of Baumgartner et al. (2). The observed cutoff value appeared to be plausible since individuals classified as low FFM also exhibited significantly lower values of muscle strength and aerobic fitness indexes. Moreover, the prevalence observed among 
older women was $19.8 \%$, in agreement with previous studies $(2,4,25)$.

Practical applications of the present investigation are easily envisioned. When performing DXA in older postmenopausal women similar to those studied here, values of height in meters and FM in kilograms should be applied in the following equation: AFFM $=-14,529+(17,989 x$ height in meters $)+(0.1307 x$ total fat mass in $\mathrm{kg})$. The result corresponds to the expected AFFM based on height and $F M$, and thus should be subtracted from the actual AFFM (i.e., the sum of arm and leg FFM measured by DXA). If the residual is equal to or below -3.4 the individual is classified as having sarcopenic obesity and is likely to exhibit low values of muscle strength and aerobic fitness. These calculations can be easily performed using software following the DXA exams and can provide assessment of FFM and FM in addition to bone mineral density calculations. The procedure would permit classifying a postmenopausal woman as having reduced FFM for her body size, leading clinically relevant follow-up.

The present study is not without limitations. The sample was composed of older Brazilian women and thus the results cannot be extrapolated to other populations or be applied

\section{References}

1. Rosenberg IH. Summary comments. Am J Clin Nutr 1989; 50: 1231-1233.

2. Baumgartner RN, Koehler KM, Gallagher D, Romero L, Heymsfield SB, Ross RR, et al. Epidemiology of sarcopenia among the elderly in New Mexico. Am J Epidemiol 1998; 147: 755-763.

3. Lima RM, Bezerra LM, Rabelo HT, Silva MA, Silva AJ, Bottaro M, et al. Fat-free mass, strength, and sarcopenia are related to bone mineral density in older women. $J$ Clin Densitom 2009; 12: 35-41.

4. Newman AB, Kupelian V, Visser M, Simonsick E, Goodpaster B, Nevitt M, et al. Sarcopenia: alternative definitions and associations with lower extremity function. J Am Geriatr Soc 2003; 51: 1602-1609.

5. Janssen I, Shepard DS, Katzmarzyk PT, Roubenoff R. The healthcare costs of sarcopenia in the United States. J Am Geriatr Soc 2004; 52: 80-85.

6. Cruz-Jentoft AJ, Baeyens JP, Bauer JM, Boirie Y, Cederholm T, Landi F, et al. Sarcopenia: European consensus on definition and diagnosis: Report of the European Working Group on Sarcopenia in Older People. Age Ageing 2010; 39: 412423.

7. Hansen RD, Raja C, Aslani A, Smith RC, Allen BJ. Determination of skeletal muscle and fat-free mass by nuclear and dual-energy $\mathrm{X}$-ray absorptiometry methods in men and women aged 51-84 y (1-3). Am J Clin Nutr 1999; 70: 228233.

8. Wang ZM, Visser M, Ma R, Baumgartner RN, Kotler D, Gallagher D, et al. Skeletal muscle mass: evaluation of neutron activation and dual-energy X-ray absorptiometry methods. J Appl Physiol 1996; 80: 824-831. to men. Moreover, the cross-sectional design of the study does not establish cause-effect relationships between sarcopenic obesity and the examined health-related variables. Finally, after this initial investigation, future studies should reproduce the observations, investigate further the potential use of the classification proposed here in clinical settings and examine its association with other clinical outcomes such as cardiovascular and metabolic risk factors.

The present study proposes an approach for identifying postmenopausal women with reduced FFM for their body size. The observed prevalence was approximately $20 \%$ and was associated with reduced muscle strength and aerobic fitness indexes. The condition of low muscle mass/high fat mass has been named sarcopenic obesity and thus the cutoff value presented here can be an option for its identification.

\section{Acknowledgments}

We thank the participants of the study. Research supported by Research Support Foundation of the Federal District (FAP-DF, \#193.000.555/2009) and by CNPq (\#301086/2007-9).
9. Zamboni M, Mazzali G, Fantin F, Rossi A, Di Francesco V. Sarcopenic obesity: a new category of obesity in the elderly. Nutr Metab Cardiovasc Dis 2008; 18: 388-395.

10. Tanko LB, Movsesyan L, Mouritzen U, Christiansen C, Svendsen OL. Appendicular lean tissue mass and the prevalence of sarcopenia among healthy women. Metabolism 2002; 51: 69-74.

11. Bouchard DR, Janssen I. Dynapenic-obesity and physical function in older adults. J Gerontol A Biol Sci Med Sci 2010; 65: 71-77.

12. Schrager MA, Metter EJ, Simonsick E, Ble A, Bandinelli S, Lauretani $F$, et al. Sarcopenic obesity and inflammation in the InCHIANTI study. J Appl Physiol 2007; 102: 919-925.

13. Jarosz PA, Bellar A. Sarcopenic obesity: an emerging cause of frailty in older adults. Geriatr Nurs 2009; 30: 64-70.

14. Bouchard DR, Dionne IJ, Brochu M. Sarcopenic/obesity and physical capacity in older men and women: data from the Nutrition as a Determinant of Successful Aging (NuAge) - The Quebec Longitudinal Study. Obesity 2009; 17: 2082-2088.

15. Cesari M, Kritchevsky SB, Baumgartner RN, Atkinson HH, Penninx BW, Lenchik L, et al. Sarcopenia, obesity, and inflammation - results from the Trial of Angiotensin Converting Enzyme Inhibition and Novel Cardiovascular Risk Factors Study. Am J Clin Nutr 2005; 82: 428-434.

16. Drouin JM, Valovich-mcLeod TC, Shultz SJ, Gansneder $\mathrm{BM}$, Perrin DH. Reliability and validity of the Biodex system 3 pro isokinetic dynamometer velocity, torque and position measurements. Eur J Appl Physiol 2004; 91: 22-29.

17. Bottaro M, Russo A, Oliveira RJ. The effects of rest interval on quadriceps torque during an isokinetic testing protocol in elderly. J Sci Med Sport 20056; 4: 285-290. 
18. Brown L. Isokinetics in human performance. 1st edn. Champaign: Human Kinetics; 2000.

19. Wasserman K, Mcllroy MB. Detecting the threshold of anaerobic metabolism in cardiac patients during exercise. Am J Cardiol 1964; 14: 844-852.

20. Yamamoto Y, Miyashita M, Hughson RL, Tamura S, Shinohara $\mathrm{M}$, Mutoh $\mathrm{Y}$. The ventilatory threshold gives maximal lactate steady state. Eur J Appl Physiol Occup Physiol 1991; 63: 55-59.

21. Oliveira RJ, Mota AM, Bottaro M, Pitanga F, Guido M, Leite TKM, et al. Association between sarcopenia-related phenotypes with aerobic capacity indexes of older women. J Sci Med Sport 2009; 8: 337-343.

22. Stenholm S, Harris TB, Rantanen T, Visser M, Kritchevsky SB, Ferrucci L. Sarcopenic obesity: definition, cause and consequences. Curr Opin Clin Nutr Metab Care 2008; 11: 693-700.

23. Zamboni M, Zoico E, Scartezzini T, Mazzali G, Tosoni P, Zivelonghi $A$, et al. Body composition changes in stable- weight elderly subjects: the effect of sex. Aging Clin Exp Res 2003; 15: 321-327.

24. Rolland Y, Lauwers-Cances V, Cristini C, Abellan Van Kan G, Janssen I, Morley JE, et al. Difficulties with physical function associated with obesity, sarcopenia, and sarcopenic-obesity in community-dwelling elderly women: the EPIDOS (EPIDemiologie de l'OSteoporose) Study. Am J Clin Nutr 2009; 89: 1895-1900.

25. Kim TN, Yang SJ, Yoo HJ, Lim KI, Kang HJ, Song W, et al. Prevalence of sarcopenia and sarcopenic obesity in Korean adults: the Korean Sarcopenic Obesity Study. Int J Obes 2009; 33: 885-892.

26. Stephen WC, Janssen I. Sarcopenic-obesity and cardiovascular disease risk in the elderly. $J$ Nutr Health Aging 2009; 13: 460-466.

27. Heber D, Ingles S, Ashley JM, Maxwell MH, Lyons RF, Elashoff RM. Clinical detection of sarcopenic obesity by bioelectrical impedance analysis. Am J Clin Nutr 1996; 64: 472S-477S. 